\title{
An efficient and facile synthesis of divergent C-3/C-5 bis-functionalized 2-oxindoles from 5-formyl-Morita-Baylis-Hillman adducts of oxindole
}

\author{
KODIRAJAN SELVAKUMAR ${ }^{\mathrm{a}, *}$, KANDAPALAM ARUN PRASATH LINGAM $^{\mathrm{b}}$, \\ RAMA VARMA LUXMI VARMA ${ }^{\mathrm{c}}$ and POOVAN SHANMUGAVELAN ${ }^{\mathrm{d}, *}$ \\ a Department of Chemistry, Thiagarajar College, Madurai 625 009, Tamil Nadu, India \\ ${ }^{\mathrm{b}}$ Department of Chemistry, Kamaraj College, Tuticorin 628 003, Tamil Nadu, India \\ ${ }^{c}$ Chemical Sciences and Technology Division, National Institute for Interdisciplinary Science and Technology, \\ Thiruvananthapuram 695 019, Kerala, India \\ ${ }^{\mathrm{d} D e p a r t m e n t ~ o f ~ C h e m i s t r y, ~ S c h o o l ~ o f ~ S c i e n c e, ~ T a m i l n a d u ~ O p e n ~ U n i v e r s i t y, ~ S a i d a p e t, ~ C h e n n a i ~} 600015$, \\ Tamil Nadu, India \\ e-mail: selvaramkumar@gmail.com; shanchemmku@gmail.com
}

MS received 27 November 2014; revised 13 May 2015; accepted 14 May 2015

\begin{abstract}
An efficient and facile synthesis of divergent C-3/C-5 bis-functionalized 2-oxindole derivatives has been achieved from 3,5-bis-Morita-Baylis-Hillman (MBH) adducts of oxindole via nucleophilic substitution reaction for the first time. Wider scope of substrate and rate acceleration has been observed in second $\mathrm{MBH}$ reaction under typical reaction condition. The synthetic usefulness of bi-functionalized bis-allyl derivative has been demonstrated by the synthesis of potent bis-pyrazole via [3+2]-annulation strategy.
\end{abstract}

Keywords. Bis-MBH adduct; Oxindole; Nucleophilic substitution; Tri-substituted olefins; bis-pyrazole.

\section{Introduction}

Oxindole and their derivatives continue to receive extensive attention in organic synthesis and also serve as potential synthons for the synthesis of alkaloids, drug intermediates and clinical pharmaceuticals. ${ }^{1}$ Aimed at that purpose, oxindole has been functionalized in many ways, namely, nucleophilic substitution at C3 carbonyl, $^{2}$ alkylation/acylation at oxindole nitrogen ${ }^{3}$ and electrophilic substitution at aromatic nuclei. ${ }^{4}$ In particular, bi-functionalization of isatin at $\mathrm{C}-2$ carbonyl (amide carbonyl) with oxindole nitrogen provided the natural products such as ophiuroidine and hydroxytryptanthrins by Prey demethylation method. ${ }^{5}$ In recent years, MBH adduct of oxindole has attracted attention due to its significance of multi-functionalized motifs, because they are also observed in a wide range of bioactive compounds and natural products. ${ }^{6}$ Owing to the lack of inherent functional entity on the aryl core of $\mathrm{MBH}$ adduct of oxindole, the synthetic transformation has been carried out preferably at C-3/ N-position. ${ }^{7}$ Interestingly, a mild and single electron oxidation strategy has been reported for the oxidation of 5-methyl

*For correspondence
$\mathrm{MBH}$ adduct of isatin into 5-formyl-mono $\mathrm{MBH}$ derivative $(\mathrm{mMBH})$, which could enable various $\mathrm{C}-\mathrm{C}, \mathrm{C}-\mathrm{O}$ and $\mathrm{C}-\mathrm{N}$ bond-forming reactions. ${ }^{8}$ Among the various carbon-carbon bond-forming reactions, $\mathrm{MBH}$ reaction plays an important role in synthetic chemistry, because they also serve as versatile synthons in the construction of complex molecular frameworks. ${ }^{9}$

In general, the MBH reaction of benzaldehyde exhibits a poor substrate scope and also requires prolonged reaction time under typical reaction conditions. To address these limitations, a number of strategies have been developed to pursue the $\mathrm{MBH}$ adduct either by increasing the concentration of reactive intermediates and/or by activating the electrophiles. ${ }^{10}$ In addition, most of the reported strategies were applicable to a few activated alkenes and some catalysts showed substrateassociated rate acceleration. ${ }^{11}$ We describe the synthesis of highly functionalized bis-allyl derivatives of oxindole from bis-MBH adducts via nucleophilic isomerization of $\mathrm{C}, \mathrm{O}$ and $\mathrm{Br}$ nucleophiles. The second $\mathrm{MBH}$ reaction of 5-formyl MBH (mMBH) adduct has displayed a broad substrate scope with a variety of activated alkenes and shows substrate-allied rate acceleration under the common catalyst of 1,4-diazabicyclo [2.2.2] octane (DABCO). The successive synthetic transformations of bis-MBH adduct provide a multi-functionalized, trisubstituted olefins and bis-pyrazole derivatives. ${ }^{12}$ 


\section{Experimental}

All chemicals and solvents were purchased from SigmaAldrich, Merck, and used without further purification. All reactions were carried out in oven-dried glassware. Progress of reactions was monitored by thin layer chromatography (TLC), while purification of crude compounds was done by column chromatography using silica gel (100-200 mesh). NMR spectra were recorded at 500 and $300 \mathrm{MHz}$ (based on availability of the instruments), 125 and $75 \mathrm{MHz}$ (for ${ }^{13} \mathrm{C}$ ), respectively, on Bruker Avance DPX-500 MHz and Bruker Avance DPX-300 MHz. Chemical shifts are reported in $\delta$ (ppm) relative to TMS $\left({ }^{1} \mathrm{H}\right)$ or $\mathrm{CDCl}_{3}\left({ }^{13} \mathrm{C}\right)$ as internal standards. The multiplicities are shown as the abbreviations: $\mathrm{s}$ (singlet), brs (broad singlet), d (doublet), t (triplet), m (multiplet). Mass spectra were recorded using JEOL JMS $600 \mathrm{H}$ mass spectrometer. Infrared spectra were determined in $0.10 \mathrm{~mm}$ matched cells with a Bomem MB series FT-IR spectrometer using a scan time of 32 min in auto-suppression and were calibrated with polystyrene film. The infrared frequencies $\left(\mathrm{cm}^{-1}\right)$ of the observed hydroxyl bands are accurate to $\pm 0.5 \mathrm{~cm}^{-1}$. Yield refers to quantities obtained after chromatography.

\subsection{General procedure for the synthesis of bis-MBH adducts $(\mathbf{3 a - m})$}

A mixture of mMBH adducts of 5-formyl- $N$-alkylisatin 1a-g (100 mg), activated alkenes $\mathbf{2 a - g}$ (1.5 equiv.) and DABCO $(20 \mathrm{~mol} \%)$ was stirred under neat condition at for 8-32 h. After completion of the reaction (monitored by TLC), the reaction mixture was diluted with ethyl acetate. The organic layer was washed successively with $0.2 \mathrm{~N} \mathrm{HCl}$, water and then brine solution. The organic layer was separated, dried over $\mathrm{Na}_{2} \mathrm{SO}_{4}$ and then concentrated in vacuo. The crude product was obtained and purified by silica gel column chromatography using EtOAc: hexane (40:60) as eluent. The desired bis-MBH adducts of oxindole (3a-m) was obtained in moderate to excellent yield (45-94\%).

\subsection{General procedure for the isomerization with oxygen and carbon nucleophiles}

A mixture of bis-MBH adduct of oxindole $3 \mathbf{b}(50 \mathrm{mg}$, $0.152 \mathrm{mmol})$, trimethylorthoformate $(2 \mathrm{~mL})$ or propargyl alcohol $(2 \mathrm{~mL})$ or $\mathrm{Ar}-\mathrm{H}(2 \mathrm{~mL})$ and montmorillonite K-10 Clay $(50 \%$ w/w) was subjected under solvolysis condition. After completion of the reaction (monitored by TLC), the reaction mixture was diluted with $\mathrm{CH}_{2} \mathrm{Cl}_{2}$ and passed through a pad of celite-545. On concentration under vacuum, the resulted crude mixture was purified by a silica gel column chromatography using EtOAc:hexane (15:85) as eluent. The isomerization afforded the corresponding isomerized products in combined moderate-to-good yield (54-75\%).

\subsection{Procedure for the isomerization of Bromide nucleophile}

A mixture of bis-MBH adduct of oxindole $\mathbf{3 b}(50 \mathrm{mg}$, $0.152 \mathrm{mmol}), 46 \%$ aqua. $\mathrm{HBr}$ ( 2 equiv.) and silica gel $(0.2 \mathrm{~g})$ are made a slurry. The slurry was subjected to microwave irradiation $(70 \%$ power level, $5 \mathrm{sec}$. pulse, SAMSUNG, Model: CE $118 \mathrm{KF}$ ) for $10 \mathrm{~min}$. The crude mixture was cooled to room temperature and then extracted with $\mathrm{CH}_{2} \mathrm{Cl}_{2}$ and the organic phase washed with water. The organic layer was separated and dried (anhyd. $\mathrm{Na}_{2} \mathrm{SO}_{4}$ ) and concentrated in vacuo. The obtained crude mixture was purified by silica gel column chromatography using a gradient elution with EtOAc:hexane (20: 80) as eluent to afford allyl bromide of oxindole derivative (4d and $\mathbf{5 d}$ ) in combined good yield (67\%).

2.4 General procedure for the synthesis of bis-pyrazole from bis-allyl bromide of oxindole derivative

A mixture bis-allyl bromide $\mathbf{5 d}(100 \mathrm{mg}, 0.221 \mathrm{mmol})$, dimethyl sulphide (1.2 equiv., $0.028 \mathrm{mmol}$ ), $\mathrm{K}_{2} \mathrm{CO}_{3}(2.1$ equiv. $64 \mathrm{mg})$ in $\mathrm{CH}_{3} \mathrm{CN}(2.0 \mathrm{~mL})$ and diethyl azodicarboxylate 6 ( 2.2 equiv.) were added successively at RT. After completion of the reaction (monitored by TLC), the solvent was removed under vacuum. Water $(5.0 \mathrm{~mL})$ was added to the residue and extracted with ether $(3 \times 5.0 \mathrm{~mL})$. Combined organic layer was dried over anhydrous $\mathrm{Na}_{2} \mathrm{SO}_{4}$ and the solvent was evaporated. The obtained crude product was purified by silica gel column chromatography using EtOAc: hexane (25:75) eluent to afford bis-pyrazole 7 in good yield (82\%).

2.4a 2-\{[3-(1-Cyano-vinyl)-3-hydroxy-1-methyl-2-oxo2,3-dihydro-1H-indol-5-yl]-hydroxy-methyl\}-acrylonitrile (3a): Gummy matter, yield $12 \%$. IR $\left(\mathrm{CH}_{2} \mathrm{Cl}_{2}\right)$ $\left(v_{\max }, \mathrm{cm}^{-1}\right)$ : 973, 1063, 1078, 1719, 2226, 2876, 3110, 3365. ${ }^{1} \mathrm{H}$ NMR $\left(300 \mathrm{MHz}, \mathrm{CDCl}_{3}\right): \delta(\mathrm{ppm}) 3.28(3 \mathrm{H}$, s), $3.84(1 \mathrm{H}, \mathrm{bs}), 4.44(1 \mathrm{H}, \mathrm{bs}), 5.33(1 \mathrm{H}, \mathrm{s}), 5.98-6.03$ $(2 \mathrm{H}, \mathrm{m}), 7.26-7.38(2 \mathrm{H}, \mathrm{m}), 7.39-7.47(3 \mathrm{H}, \mathrm{m}) .{ }^{13} \mathrm{C}$ NMR $\left(75 \mathrm{MHz}, \mathrm{CDCl}_{3}\right): \delta(\mathrm{ppm})$ 27.2, 62.9, 73.4, 109.4, 122.0, 123.2, 123.4, 125.0, 125.1, 126.0, 126.2, 129.4, 129.6, 130.3, 130.5, 173.8. FAB mass: 296.43 $(\mathrm{M}+1)$. Anal. Calcd. for $\mathrm{C}_{16} \mathrm{H}_{13} \mathrm{~N}_{3} \mathrm{O}_{3}$ : C, 65.08; $\mathrm{H}$, 4.44; N, 14.23; Found: C, 65.05; H, 4.42; N, 14.25.

2.4b 2-[5-(2-Cyano-1-hydroxy-allyl)-3-hydroxy-1methyl-2-oxo-2,3-dihydro-1H-indol-3-yl]-acrylic acid 
methyl ester (3b): Gummy matter, yield 87\%. IR $\left(\mathrm{CH}_{2} \mathrm{Cl}_{2}\right)\left(v_{\max }, \mathrm{cm}^{-1}\right): 1053,1089,1643,1716,2230$, 3088, 3382. ${ }^{1} \mathrm{H}$ NMR $\left(300 \mathrm{MHz}, \mathrm{CDCl}_{3}\right): \delta(\mathrm{ppm})$ $3.20(3 \mathrm{H}, \mathrm{s}), 3.60(3 \mathrm{H}, \mathrm{s}), 4.24-4.28(1 \mathrm{H}, \mathrm{bs}), 5.18(1 \mathrm{H}$, s), 5.98-5.99(1H, s), 6.06-6.10(1H, d, $J=8.0 \mathrm{~Hz})$, 6.37-6.38(1H, s), 6.53(1H, s), 6.82-6.85(d, $1 \mathrm{H}, J=$ $8.0 \mathrm{~Hz}), 7.25(1 \mathrm{H}, \mathrm{s}), 5.18(1 \mathrm{H}, \mathrm{s}), 7.34(1 \mathrm{H}, \mathrm{s}) .{ }^{13} \mathrm{C}$ NMR (75 MHz, $\left.\mathrm{CDCl}_{3}\right): \delta$ (ppm) 26.5, 52.0, 60.4, 73.6, 108.7, 121.9, 122.2, 128.2, 128.6, 128.8, 129.8, 129.9, 130.0, 134.2, 134.2, 164.6, 176.3. FAB mass: 329.45 $(\mathrm{M}+1)$. Anal. Calcd. for $\mathrm{C}_{17} \mathrm{H}_{16} \mathrm{~N}_{2} \mathrm{O}_{5}$ : C, 62.19; H, $4.91 ;$ N, 8.53. Found: C, 62.21; H, 4.90; N, 8.54.

\section{4c 2-\{[3-(1-Cyano-vinyl)-3-hydroxy-2-oxo-1-prop-} 2-ynyl-2,3-dihydro-1H-indol-5-yl]-hydroxy-methyl\}acrylonitrile $(3 \mathrm{c})$ : Gummy matter, yield $74 \%$. IR $\left(\mathrm{CH}_{2} \mathrm{Cl}_{2}\right)\left(v_{\max }, \mathrm{cm}^{-1}\right): 1069,1627,1718,2218,3363$. ${ }^{1} \mathrm{H}$ NMR $\left(300 \mathrm{MHz}, \mathrm{CDCl}_{3}\right): \delta(\mathrm{ppm}) 2.31(1 \mathrm{H}, \mathrm{s})$, 3.02 $(1 \mathrm{H}, \mathrm{bs}), 4.51(2 \mathrm{H}, \mathrm{s}) 4.54(1 \mathrm{H}, \mathrm{bs}), 5.33(1 \mathrm{H}, \mathrm{s})$, 6.06-6.35(4H, m), 7.14(1H, s), 7.46-7.51(2H, m). ${ }^{13} \mathrm{C}$ NMR (75 MHz, $\mathrm{CDCl}_{3}$ ): $\delta$ (ppm) 29.9, 60.1, 73.3, 73.7, $75.5,110.4,113.4,115.0,116.4,123.0,123.2$, 126.1, 127.7, 129.5, 129.8, 131.7, 142.1, 172.9. FAB mass: $320.13(\mathrm{M}+1)$. Anal. Calcd. for $\mathrm{C}_{18} \mathrm{H}_{13} \mathrm{~N}_{3} \mathrm{O}_{3}: \mathrm{C}, 67.71$; H, 4.10; N, 13.16. Found: C, 67.74; H, 4.09; N, 13.17.

2.4d 2-[5-(2-Cyano-1-hydroxy-allyl)-3-hydroxy-2-oxo1-prop-2-ynyl-2,3-dihydro-1H-indol-3-yl]-acrylic acid methyl ester $(\mathbf{3 d})$ : Gummy matter, yield $83 \%$. IR $\left(\mathrm{CH}_{2} \mathrm{Cl}_{2}\right)\left(v_{\max }, \mathrm{cm}^{-1}\right): 1660,1713,1726,2216,2225$, 3389. ${ }^{1} \mathrm{H}$ NMR $\left(300 \mathrm{MHz}, \mathrm{CDCl}_{3}\right.$ ): $\delta$ (ppm) 2.27-2.30 $(1 \mathrm{H}, \mathrm{s}), 3.51(1 \mathrm{H}, \mathrm{bs}), 3.57(3 \mathrm{H}, \mathrm{s}), 4.39-4.42(1 \mathrm{H}, \mathrm{bs})$, $4.48(2 \mathrm{H}, \mathrm{s}), 5.20(1 \mathrm{H}, \mathrm{s}), 5.98-6.57(4 \mathrm{H}, \mathrm{m}), 7.06-$ $7.09(1 \mathrm{H} . \mathrm{d}, J=8.0 \mathrm{~Hz}), 7.18(1 \mathrm{H}, \mathrm{s}), 7.37-7.43(1 \mathrm{H}$, $\mathrm{d}, J=8.0 \mathrm{~Hz}) \cdot{ }^{13} \mathrm{C}$ NMR $\left(75 \mathrm{MHz}, \mathrm{CDCl}_{3}\right): \delta(\mathrm{ppm})$ 29.7, 52.1, 60.40, 72.8, 73.8, 76.1, 109.9, 122.5, 126.4, 128.2, 128.7, 128.9, 129.6, 129.8, 134.8, 138.8, 143.3, 165.1, 175.1. FAB mass: $352.37\left(\mathrm{M}^{+}\right)$. Anal. Calcd. for $\mathrm{C}_{19} \mathrm{H}_{16} \mathrm{~N}_{2} \mathrm{O}_{5}$ : C, 64.77; H, 4.58; N, 7.95. Found: C, 64.79; H, 4.57; N, 7.97.

2.4e 2-\{[1-Benzyl-3-(1-cyano-vinyl)-3-hydroxy-2-oxo2,3-dihydro-1H-indol-5-yl]-hydroxy-methyl\}-acrylonitrile (3e): Gummy matter, yield $79 \%$. IR $\left(\mathrm{CH}_{2} \mathrm{Cl}_{2}\right)$ $\left(v_{\max }, \mathrm{cm}^{-1}\right): 1079,1717,2236,3108,3371 .{ }^{1} \mathrm{H}$ NMR $\left(300 \mathrm{MHz}, \mathrm{CDCl}_{3}\right): \delta(\mathrm{ppm}) 2.55(1 \mathrm{H}, \mathrm{bs}), 4.01(1 \mathrm{H}$, bs), $4.65-4.70(1 \mathrm{H}, \mathrm{d}, J=15.7 \mathrm{~Hz}), 4.91-4.97(\mathrm{~d}, 1 \mathrm{H}$, $J=5.7 \mathrm{~Hz},), 5.22(1 \mathrm{H}, \mathrm{s}), 5.88-6.26(4 \mathrm{H}, \mathrm{m}), 6.68-$ $6.71(1 \mathrm{H}, \mathrm{d}, J=8.1 \mathrm{~Hz}), 7.24-7.27(6 \mathrm{H}, \mathrm{m}), 7.36-7.40$ $(1 \mathrm{H}, \mathrm{d}, J=8.1 \mathrm{~Hz}) \cdot{ }^{13} \mathrm{C} \mathrm{NMR}\left(75 \mathrm{MHz}, \mathrm{CDCl}_{3}\right): \delta$ (ppm) 42.6, 62.1, 73.5, 115.6, 116.6, 116.87, 125.6, 125.6, 126.1, 126.7, 127.3, 127.7, 127.9, 128.6, 128.8, $129.8,129.9,130.5,134.3,135.2,135.39,174.3$. FAB mass: $372.41(\mathrm{M}+1)$. Anal. Calcd. for $\mathrm{C}_{22} \mathrm{H}_{17} \mathrm{~N}_{3} \mathrm{O}_{3}$ : C, 71.15; H, 4.61; N, 11.31. Found: C, 71.12; H, 4.60; N, 11.32 .

2.4f 2-[1-Benzyl-5-(2-cyano-1-hydroxy-allyl)-3-hydroxy2-oxo-2,3-dihydro-1H-indol-3-yl]-acrylic acid methyl ester (3f): Gummy matter, yield 88\%. IR $\left(\mathrm{CH}_{2} \mathrm{Cl}_{2}\right)$ $\left(v_{\max }, \mathrm{cm}^{-1}\right) 1181,1610,1721,2211,2990,3295 .{ }^{1} \mathrm{H}$ NMR (300 MHz, $\left.\mathrm{CDCl}_{3}\right): \delta(\mathrm{ppm}) 3.11(1 \mathrm{H}, \mathrm{bs}), 3.62$ $(3 \mathrm{H}, \mathrm{s}), 3.64(1 \mathrm{H}, \mathrm{bs}), 4.79-4.84(1 \mathrm{H}, \mathrm{d}, J=17.5 \mathrm{~Hz})$, $4.90-4.95(1 \mathrm{H}, \mathrm{d}, J=17.5 \mathrm{~Hz}), 5.1(1 \mathrm{H}, \mathrm{s}), 5.94-6.61$ $(4 \mathrm{H}, \mathrm{m}), 6.67-6.70(1 \mathrm{H}, \mathrm{d}, J=8.2 \mathrm{~Hz}), 7.17(1 \mathrm{H}, \mathrm{s})$, 7.27-7.36(6H,m). ${ }^{13} \mathrm{C} \mathrm{NMR}\left(75 \mathrm{MHz}, \mathrm{CDCl}_{3}\right): \delta(\mathrm{ppm})$ 44.1, 52.0, 73.7, 76.0, 109.8, 121.2, 122.2, 125.8, 125.9, 127.3, 127.7, 128.5, 128.6, 128.73, 128.8, 129.8, 129.9, $130.1,134.2,135.1,138.5,165.0,176.4$. FAB mass: 405.76 (M+1). Anal. Calcd. for $\mathrm{C}_{23} \mathrm{H}_{20} \mathrm{~N}_{2} \mathrm{O}_{5}$ : C, 68.31; H, 4.98; N, 6.93. Found: C, 68.34; H, 4.97; N, 6.95.

2.4g 2-\{Hydroxy-[3-hydroxy-3-(1-methoxycarbonylvinyl)-1-methyl-2-oxo-2,3-dihydro-1H-indol-5-yll-methyl\}acrylic acid methyl ester (3h): Gummy matter, yield 94\%. IR $\left(\mathrm{CH}_{2} \mathrm{Cl}_{2}\right)\left(v_{\max }, \mathrm{cm}^{-1}\right): 1254,1631,1713$, 1718, 3215, 3391. ${ }^{1} \mathrm{H}$ NMR $\left(300 \mathrm{MHz}, \mathrm{CDCl}_{3}\right): \delta$ (ppm) 3.20 (s, 3H), $3.40(1 \mathrm{H}, \mathrm{bs}), 3.58(3 \mathrm{H}, \mathrm{s}), 3.66$ $(3 \mathrm{H}, \mathrm{s}), 4.46(1 \mathrm{H}, \mathrm{bs}), 5.46(1 \mathrm{H}, \mathrm{s}), 5.79-5.84(1 \mathrm{H}, \mathrm{m})$, $6.28-6.29(1 \mathrm{H}, \mathrm{d}, J=6 \mathrm{~Hz}), 6.43(1 \mathrm{H}, \mathrm{s}), 6.54(1 \mathrm{H}, \mathrm{s})$, 6.78-6.81 (1H, m), 7.18 (1H, s), 7.26-7.27 (1H, m). ${ }^{13} \mathrm{C}$ NMR (75 MHz, $\left.\mathrm{CDCl}_{3}\right): \delta(\mathrm{ppm}) 26.4,51.9,52.0,72.6$, 76.0, 108.4, 122.4, 125.9, 128.0, 128.6, 128.7, 129.6, 136.1, 138.9, 141.8, 165.0, 166.6, 176.4. FAB mass: $362.16(\mathrm{M}+1)$. Anal. Calcd. for $\mathrm{C}_{18} \mathrm{H}_{19} \mathrm{NO}_{7}$ : C, 59.83; H, 5.30; N, 3.88; Found: C, 59.84; H, 5.31; N, 3.87 .

2.4h 2-\{Hydroxy-[3-hydroxy-3-(1-methoxycarbonylvinyl)-1-methyl-2-oxo-2,3-dihydro-1H-indol-5-yl]-methyl\}acrylic acidbutylester (3i): Gummy matter, yield 54\%. IR $\left(\mathrm{CH}_{2} \mathrm{Cl}_{2}\right)\left(v_{\max }, \mathrm{cm}^{-1}\right): 1184,1589,1720,3020$, 3296. ${ }^{1} \mathrm{H}$ NMR $\left(300 \mathrm{MHz}, \mathrm{CDCl}_{3}\right): \delta$ (ppm) 0.89$0.92(3 \mathrm{H}, \mathrm{m}), 1.33-1.34(2 \mathrm{H}, \mathrm{m}), 3.24(3 \mathrm{H}, \mathrm{s}), 3.25$ $(1 \mathrm{H}, \mathrm{bs}), 3.34(2 \mathrm{H}, \mathrm{m}), 3.65(3 \mathrm{H}, \mathrm{s}), 4.10-4.11(2 \mathrm{H}$, m), $4.87(1 \mathrm{H}, \mathrm{bs}), 5.50(1 \mathrm{H}, \mathrm{s}), 5.75-5.79(1 \mathrm{H}, \mathrm{d}$, $J=17.5 \mathrm{~Hz}), 6.30-6.36(1 \mathrm{H}, \mathrm{m}), 6.40(1 \mathrm{H}, \mathrm{s}), 6.56$ $(1 \mathrm{H}, \mathrm{s}), 6.82-6.85(1 \mathrm{H}, \mathrm{m}), 7.18(1 \mathrm{H}, \mathrm{s}), 7.31-7.39$ $(1 \mathrm{H}, \mathrm{m}) .{ }^{13} \mathrm{C} \mathrm{NMR}\left(75 \mathrm{MHz}, \mathrm{CDCl}_{3}\right): \delta(\mathrm{ppm}) 13.6$, 19.1, 26.5, 30.5, 52.0, 69.0, 73.0, 108.5, 122.6, 125.9, 126.0, 127.8, 127.8, 128.6, 128.8, 129.3, 139.0, 165.1, 170.5, 176.2. FAB mass: $404.47(\mathrm{M}+1)$. Anal. Calcd. for $\mathrm{C}_{21} \mathrm{H}_{25} \mathrm{NO}_{7}$ : C, 62.52; H, 6.25; N, 3.47. Found: C, $62.50 ; \mathrm{H}, 6.26 ; \mathrm{N}, 3.48$.

2.4i 2-(5-Formyl-3-hydroxy-1-methyl-2-oxo-2,3-dihydro1H-indol-3-yl)-4-methylene-5-oxo-hexanoic acid methyl 
ester (3j): Gummy matter, yield 54\%. IR $\left(\mathrm{CH}_{2} \mathrm{Cl}_{2}\right)$ $\left(v_{\max }, \mathrm{cm}^{-1}\right): 1056,1574,1681,1716,1715,1726$, 3315. ${ }^{1} \mathrm{H}$ NMR $\left(300 \mathrm{MHz}, \mathrm{CDCl}_{3}\right): \delta(\mathrm{ppm}) 2.31(3 \mathrm{H}$, s), 2.67-2.72 $(1 \mathrm{H}, \mathrm{dd}, J=2.5,10.5 \mathrm{~Hz}), 2.98-3.01$ $(1 \mathrm{H}, \mathrm{dd}, J=1.5,10.5 \mathrm{~Hz}), 3.23(3 \mathrm{H}, 2), 3.57(3 \mathrm{H}, \mathrm{s})$, $3.65(1 \mathrm{H}, \mathrm{m}), 4.86(1 \mathrm{H}, \mathrm{bs}), 5.82-6.07(2 \mathrm{H}, \mathrm{m}), 6.96$ $(1 \mathrm{H}, \mathrm{s}), 7.88-8.02(2 \mathrm{H}, \mathrm{m}), 9.91(1 \mathrm{H}, \mathrm{s}) .{ }^{13} \mathrm{C} \mathrm{NMR}$ $\left(75 \mathrm{MHz}, \mathrm{CDCl}_{3}\right): \delta$ (ppm) 25.6, 26.5, 27.5, 50.3, 52.0, 75.5, 108.7, 126.3, 127.9, 128.6, 129.2, 132.0, 134.0, 145.65, 171.9, 176.5, 190.6, 199.8. FAB mass: 345.51 $(\mathrm{M}+1)$. Anal. Calcd. for $\mathrm{C}_{18} \mathrm{H}_{19} \mathrm{NO}: \mathrm{C}, 62.60 ; \mathrm{H}, 5.55$; $\mathrm{N}, 4.06$. Found: C, 62.62; H, 5.54; N, 4.05.

2.4j 2-\{3-Hydroxy-5-[hydroxy-(5-oxo-cyclopent-1-enyl)methyl]-1-methyl-2-oxo-2,3-dihydro-1H-indol-3-yl\}-acrylic acid methyl ester (3k): Gummy matter, yield 89\%. IR $\left(\mathrm{CH}_{2} \mathrm{Cl}_{2}\right)\left(v_{\max }, \mathrm{cm}^{-1}\right): 1289,1615,1714,1723,3009$, 3289. ${ }^{1} \mathrm{H}$ NMR $\left(300 \mathrm{MHz}, \mathrm{CDCl}_{3}\right): \delta$ (ppm) 2.362.38 $(2 \mathrm{H}, \mathrm{m}), 2.41-2.44(2 \mathrm{H}, \mathrm{m}), 3.23(3 \mathrm{H}, \mathrm{s}), 3.47(1 \mathrm{H}$, bs), $3.53(3 \mathrm{H}, \mathrm{s}), 3.90(1 \mathrm{H}, \mathrm{bs}), 5.49(1 \mathrm{H}, \mathrm{s}), 6.41(1 \mathrm{H}$, $\mathrm{d}, J=6.5 \mathrm{~Hz}), 6.55(1 \mathrm{H}, \mathrm{s}), 6.66-6.68(1 \mathrm{H}, \mathrm{m}), 6.80$ 6.6.85(1H, m), 7.25(1H, s), 7.35-7.37(1H, t, $J=$ $6.5 \mathrm{~Hz}) .{ }^{13} \mathrm{C} \mathrm{NMR}\left(75 \mathrm{MHz}, \mathrm{CDCl}_{3}\right): \delta(\mathrm{ppm}) 22.4$, 25.7, 38.5, 52.0, 72.1, 76.2, 108.4, 122.5, 127.9, 128.3, 128.7, 129.2, 136.4, 136.6, 139.0, 140.9, 165.1, 176.2, 199.5. FAB mass: $358.46(\mathrm{M}+1)$. Anal. Calcd. for $\mathrm{C}_{19} \mathrm{H}_{19} \mathrm{NO}_{6}$ : C, 63.86; H, 5.36; N, 3.92. Found: C, 63.89; H, 5.35; N, 3.93.

2.4k 2-\{3-Hydroxy-5-[hydroxy-(6-oxo-cyclohex-1-enyl)methyl]-1-methyl-2-oxo-2,3-dihydro-1H-indol-3-yl\}-acrylic acid methyl ester (3l): Gummy matter, yield 75\%. IR $\left(\mathrm{CH}_{2} \mathrm{Cl}_{2}\right)\left(v_{\max }, \mathrm{cm}^{-1}\right): 1258,1659,1711,1725,1986$, 3310. ${ }^{1} \mathrm{H}$ NMR $\left(300 \mathrm{MHz}, \mathrm{CDCl}_{3}\right)$ : $\delta$ (ppm) 1.961.99 $(2 \mathrm{H}, \mathrm{m}), 2.36-2.38(2 \mathrm{H}, \mathrm{m}), 2.41-2.44(2 \mathrm{H}, \mathrm{m})$, $3.24(3 \mathrm{H}, \mathrm{s}), 3.41(1 \mathrm{H}, \mathrm{bs}), 3.62(3 \mathrm{H}, \mathrm{s}), 3.91(1 \mathrm{H}, \mathrm{bs})$, $5.49(1 \mathrm{H}, \mathrm{s}), 6.41(1 \mathrm{H}, \mathrm{s}), 6.55(1 \mathrm{H}, \mathrm{s}), 6.68-6.72(1 \mathrm{H}, \mathrm{m})$, 6.81-6.84(1H, m), 7.25-7.27(1H, m), 7.35-7.37(1H, m). ${ }^{13} \mathrm{C}$ NMR (75 MHz, $\mathrm{CDCl}_{3}$ ): $\delta$ (ppm) 22.4, 25.7, 26.5, 38.5, 52.0, 72.1, 76.2, 108.4, 122.5, 127.9, 128.3, 128.7, 129.2, 136.4, 136.6, 138.9, 140.9, 165.1, 176.2, 200.5. FAB mass: $372.12(\mathrm{M}+1)$. Anal. Calcd. for $\mathrm{C}_{20} \mathrm{H}_{21} \mathrm{NO}_{6}: \mathrm{C}, 64.68 ; \mathrm{H}, 5.70 ; \mathrm{N}, 3.77$. Found: $\mathrm{C}$, 64.66; H, 5.71; N, 3.78 .

2.41 2-\{3-Hydroxy-5-[hydroxy-(7-oxo-cyclohept-1-enyl)methyl]-1-methyl-2-oxo-2,3-dihydro-1H-indol-3-yl\}-acrylic acid methyl ester (3m): Gummy matter, yield 67\%. IR $\left(\mathrm{CH}_{2} \mathrm{Cl}_{2}\right)\left(v_{\max }, \mathrm{cm}^{-1}\right): 1053,1435,1713,1720,2995$, 3269. ${ }^{1} \mathrm{H}$ NMR $\left(300 \mathrm{MHz}, \mathrm{CDCl}_{3}\right)$ : $\delta$ (ppm) 1.74$1.75(4 \mathrm{H}, \mathrm{m}), 2.42(2 \mathrm{H}, \mathrm{m}), 2.50-2.51(2 \mathrm{H}, \mathrm{m}), 3.23(1 \mathrm{H}$, bs), 3.61(3H, s), 3.68(1H, bs $), 4.02(1 \mathrm{H}, \mathrm{bs}), 5.30(1 \mathrm{H}$, s), 6.41(1H, s), 6.54-6.60(2H, m), 6.79-6.81(2H, m),
7.12(1H, m), 7.22-7.25(1H, m), 7.26-7.27(1H m). ${ }^{13} \mathrm{C} \mathrm{NMR}\left(75 \mathrm{MHz}, \mathrm{CDCl}_{3}\right): \delta$ (ppm) 21.4, 24.9, 26.5, 27.6, 43.1, 52.0, 75.05, 76.1, 108.4, 121.9, 122.2, $127.8,128.1,129.3,129.3,137.2,143.7,143.8,165.1$, 176.3, 206.4. FAB mass: $386.52(\mathrm{M}+1)$. Anal. Calcd. for $\mathrm{C}_{21} \mathrm{H}_{23} \mathrm{NO}_{6} \mathrm{C}, 65.44 ; \mathrm{H}, 6.02 ; \mathrm{N}, 3.63$;. Found: $\mathrm{C}$, $65.41 ; \mathrm{H}, 6.01 ; \mathrm{N}, 3.64$.

2.5a 2-[5-(2-Cyano-3-methoxy-propenyl)-3-hydroxy1-methyl-2-oxo-2,3-dihydro-1H-indol-3-yl]-acrylic acid methylester $(4 a)$ : Gummy matter, yield $46 \%$. IR $\left(\mathrm{CH}_{2} \mathrm{Cl}_{2}\right)$ $\left(v_{\max }, \mathrm{cm}^{-1}\right): 1097,1216,1353,1634,1717,1736$, 2231，3056, 3364. ${ }^{1} \mathrm{H}$ NMR $\left(300 \mathrm{MHz}, \mathrm{CDCl}_{3}\right): \delta$ (ppm) $3.26(3 \mathrm{H}, \mathrm{s}), 3.56(3 \mathrm{H}, \mathrm{s}), 3.95(3 \mathrm{H}, \mathrm{s}) 4.10(1 \mathrm{H}$, bs $), \quad 4.22(2 \mathrm{H}, \mathrm{s}), \quad 6.28(1 \mathrm{H}, \mathrm{s}), \quad 6.62(1 \mathrm{H}, \mathrm{s}), 6.85-$ $6.88(1 \mathrm{H}, \mathrm{d}, J=8.3 \mathrm{~Hz}), 7.02(1 \mathrm{H}, \mathrm{s}), 7.79-7.82(1 \mathrm{H}, \mathrm{d}$, $J=8.3 \mathrm{~Hz}), 8.05(1 \mathrm{H}, \mathrm{s}) .{ }^{13} \mathrm{C} \mathrm{NMR}\left(75 \mathrm{MHz}, \mathrm{CDCl}_{3}\right)$ : $\delta(\mathrm{ppm}) 29.7,35.6,42.0,52.2,73.0,124.4,124.9$, $127.3,128.2,128.3,128.5,128.9,128.9,129.0,129.75$, 131.25, 164.9, 175.1. FAB mass: $343.67(\mathrm{M}+1)$. Anal. Calcd. for $\mathrm{C}_{18} \mathrm{H}_{18} \mathrm{~N}_{2} \mathrm{O}_{5}: C, 63.15 ; \mathrm{H}, 5.30 ; \mathrm{N}, 8.18$; Found: C, 63.17; H, 5.31; N, 8.17.

$2.5 b \quad 2-[5-(2-C y a n o-3-p h e n y l-p r o p e n y l)-3-h y d r o x y-1-$ methyl-2-oxo-2,3-dihydro-1H-indol-3-yl]-acrylic acid methyl ester (4b): Gummy matter, yield 53\%. IR $\left(\mathrm{CH}_{2} \mathrm{Cl}_{2}\right)\left(v_{\max }, \mathrm{cm}^{-1}\right): 1056,1333,1654,1718,2229$, 3012, 3098, 3352. ${ }^{1} \mathrm{H}$ NMR $\left(300 \mathrm{MHz}, \mathrm{CDCl}_{3}\right): \delta$ (ppm) $3.35(3 \mathrm{H}, \mathrm{s}), 3.64(4 \mathrm{H}, \mathrm{bs}), 3.66(2 \mathrm{H}, \mathrm{s}), 6.45(1 \mathrm{H}$, s), 6.58(1H, s), 6.85-6.90(3H, m), 7.26-7.35(4H, m), $7.78(1 \mathrm{H}, \mathrm{s}), 7.80-7.97(1 \mathrm{H}, \mathrm{m}) .{ }^{13} \mathrm{C}$ NMR $(75 \mathrm{MHz}$, $\mathrm{CDCl}_{3}$ ): $\delta$ (ppm) 25.4, 36.5, 54.7, 73.5, 125.4, 126.9, $126.9,127.1,127.8,128.0,128.15,128.5,128.9,129.0$, 129.1, 129.6, 132.0, 132.1, 134.0, 143.5, 144.4, 166.9, 174.3. FAB mass: $388.49(\mathrm{M}+1)$. Anal. Calcd. for $\mathrm{C}_{23} \mathrm{H}_{20} \mathrm{~N}_{2} \mathrm{O}_{4}$ : C, 71.12; H, 5.19; N, 7.21. Found: C, 71.14; H, 5.18; N, 7.20.

$2.5 \mathrm{c}$ 2-[5-(2-Cyano-3-prop-2-ynyloxy-propenyl)-3hydroxy-1-methyl-2-oxo-2,3-dihydro-1H-indol-3-yl]acrylic acid methyl ester (4c): Gummy matter, yield 54\%. IR $\left(\mathrm{CH}_{2} \mathrm{Cl}_{2}\right)\left(v_{\max }, \mathrm{cm}^{-1}\right): 1045,1098,1337,1624$, 1716, 1735, 2185, 2236, 3362. ${ }^{1} \mathrm{H}$ NMR $(300 \mathrm{MHz}$, $\left.\mathrm{CDCl}_{3}\right): \delta(\mathrm{ppm}) 2.46(1 \mathrm{H}, \mathrm{s}), 3.28(3 \mathrm{H}, \mathrm{s}), 3.65(3 \mathrm{H}, \mathrm{s})$ 3.76(1H, bs $), 4.23-4.28(4 \mathrm{H}, \mathrm{m}), 6.48(1 \mathrm{H}, \mathrm{s}), 6.58(1 \mathrm{H}$, s), $6.90-6.93(1 \mathrm{H}, \mathrm{d}, J=8.1 \mathrm{~Hz}), 7.08(1 \mathrm{H}, \mathrm{s}), 7.58(1 \mathrm{H}$, s), $7.83-7.86(1 \mathrm{H}, \mathrm{d}, J=8.1 \mathrm{~Hz}) .{ }^{13} \mathrm{C}$ NMR $(75 \mathrm{MHz}$, $\left.\mathrm{CDCl}_{3}\right): \delta$ (ppm) 22.6, 33.4, 33.5, 54.4, 65.0, 73.0, 76.0, 104.9, 112.6, 125.2, 128.2, 128.3, 130.0, 130.0, 131.7, 132.5, 138.4, 138.7, 164.0, 175.1. FAB mass: 367.65 (M+1). Anal. Calcd. for $\mathrm{C}_{20} \mathrm{H}_{18} \mathrm{~N}_{2} \mathrm{O}_{5}$ : C, 65.57; H, 4.95; N, 7.65. Found: C, 65.55; H, 4.96; N, 7.645. 
2.5d 2-[5-(3-Bromo-2-cyano-propenyl)-3-hydroxy-1methyl-2-oxo-2,3-dihydro-1H-indol-3-yl]-acrylic acid methyl ester (4d): Gummy matter, yield 43\%. IR $\left(\mathrm{CH}_{2} \mathrm{Cl}_{2}\right)$ $\left(v_{\max }, \mathrm{cm}^{-1}\right): 1223,1623,1664,1716,2258,3361 .{ }^{1} \mathrm{H}$ NMR $\left(300 \mathrm{MHz}, \mathrm{CDCl}_{3}\right): \delta(\mathrm{ppm}) 3.31(3 \mathrm{H}, \mathrm{s}), 3.41$ $(1 \mathrm{H}, \mathrm{bs}), 3.85(3 \mathrm{H}, \mathrm{s}), 4.75(2 \mathrm{H}, \mathrm{s}), 6.01-6.05(2 \mathrm{H}, \mathrm{m})$, 6.98-7.00(2H, m), 7.38-7.40(1H, d, $J=6 \mathrm{~Hz}), 7.46-$ 7.48(1H, m). ${ }^{13} \mathrm{C}$ NMR $\left(75 \mathrm{MHz}, \mathrm{CDCl}_{3}\right): \delta(\mathrm{ppm}) 23.0$, 43.4, 51.0, 75.0, 108.6, 122.3, 122.4, 127.0, 128.6, 129.0, 129.1, 131.5, 137.9, 138.2, 164.0, 175.4. FAB mass: 390.57( $\mathrm{M}+1)$. Anal. Calcd. for $\mathrm{C}_{17} \mathrm{H}_{15} \mathrm{BrN}_{2} \mathrm{O}_{4}: \mathrm{C}, 52.19$; H, 3.86; N, 7.16. Found: C, 52.18; H, 3.87; N, 7.15.

$2.5 \mathrm{e} \quad 2-[5-(2-C y a n o-3-m e t h o x y-p r o p e n y l)-1-m e t h y l-$ 2-oxo-1,2-dihydro-indol-3-ylidene]-3-methoxy-propionic acid methyl ester (5a): Gummy matter, yield 23\%. IR $\left(\mathrm{CH}_{2} \mathrm{Cl}_{2}\right)\left(v_{\max }, \mathrm{cm}^{-1}\right): 1094,1303,1609,1654,1716$, 1728, 2238, 3076, 3106. ${ }^{1} \mathrm{H}$ NMR (300 MHz, $\mathrm{CDCl}_{3}$ ): $\delta(\mathrm{ppm}) 3.33(3 \mathrm{H}, \mathrm{s}), 3.38(3 \mathrm{H}, \mathrm{s}), 4.17(3 \mathrm{H}, \mathrm{s}) 4.19(2 \mathrm{H}$, s), $4.60(3 \mathrm{H}$, s) $5.09(2 \mathrm{H}, \mathrm{s}), 6.62-6.64(1 \mathrm{H}, \mathrm{d}, J=$ $7.3 \mathrm{~Hz}), 6.87-6.90(1 \mathrm{H}, \mathrm{d}, J=8.2 \mathrm{~Hz}), 7.73(1 \mathrm{H}, \mathrm{s})$, $7.80-7.83(1 \mathrm{H}, \mathrm{d}, J=8.2 \mathrm{~Hz}) .{ }^{13} \mathrm{C}$ NMR $(75 \mathrm{MHz}$, $\left.\mathrm{CDCl}_{3}\right): \delta$ (ppm) 26.1, 45.3, 51.6, 51.9, 55.0, 59.1, 126.6, 127.2, 127.6, 128.1, 128.3, 128.5, 128.9, 129.8, 131.4, 133.8, 135.8, 158.3, 166.3. FAB mass: 356.60 $(\mathrm{M}+1)$. Anal. Calcd. for $\mathrm{C}_{19} \mathrm{H}_{20} \mathrm{~N}_{2} \mathrm{O}_{5}: \mathrm{C}, 64.04 ; \mathrm{H}$, 5.66; N, 7.86. Found: C, 64.06; H, 5.65; N, 7.85.

$2.5 f \quad 2-[5-(2-C y a n o-3-p h e n y l-p r o p e n y l)-1-m e t h y l-2-$ oxo-1,2-dihydro-indol-3-ylidene]-3-phenyl-propionic acid methyl ester (5b ): Gummy matter, yield 22\%. IR $\left(\mathrm{CH}_{2} \mathrm{Cl}_{2}\right)\left(v_{\max }, \mathrm{cm}^{-1}\right): 1053,1123,1523,1664,1713$, 1732, 2238, 3021, 3087. ${ }^{1} \mathrm{H}$ NMR $\left(300 \mathrm{MHz}, \mathrm{CDCl}_{3}\right)$ : $\delta(\mathrm{ppm}) 3.28(3 \mathrm{H}, \mathrm{s}), 3.67(2 \mathrm{H}, \mathrm{s}), 3.78(3 \mathrm{H}, \mathrm{s}) 4.58(2 \mathrm{H}$, s), $6.82-6.85(1 \mathrm{H}, \mathrm{d}, J=8.9 \mathrm{~Hz}), 7.28-7.37(12 \mathrm{H}, \mathrm{m})$, $7.68-7.71(1 \mathrm{H}, \mathrm{d}, J=8.9 \mathrm{~Hz}) .{ }^{13} \mathrm{C} \mathrm{NMR}(75 \mathrm{MHz}$, $\mathrm{CDCl}_{3}$ ): $\delta$ (ppm) 23.3, 44.7, 44.7, 53.5, 107.2, 110.6, 110.7, 115.4, 117.6, 122.4, 125.4, 126.9, 126.9, 127.1, 127.8, 128.0, 128.1, 128.5, 128.9, 129.0, 129.1, 129.64, 132.0, 132.1, 134.0, 143.5, 144.4, 165.4, 174.3. FAB mass: $448.64(\mathrm{M}+1)$. Anal. Calcd. for $\mathrm{C}_{29} \mathrm{H}_{24} \mathrm{~N}_{2} \mathrm{O}_{3}: \mathrm{C}$, 77.66; H, 5.39; N, 6.25. Found: C, 77.68; H, 5.38; N, 6.26 .

2.5g 3-Bromo-2-[5-(3-bromo-2-cyano-propenyl)-1-methyl2-oxo-1,2-dihydro-indol-3-ylidenel-propionic acid methyl ester (5d): Gummy matter, yield $24 \%$. IR $\left(\mathrm{CH}_{2} \mathrm{Cl}_{2}\right)\left(v_{\max }\right.$, $\left.\mathrm{cm}^{-1}\right): 1609,1654,1720,2260,3061 .{ }^{1} \mathrm{H}$ NMR $\left(300 \mathrm{MHz}, \mathrm{CDCl}_{3}\right): \delta(\mathrm{ppm}) 3.30(3 \mathrm{H}, \mathrm{s}), 4.10(3 \mathrm{H}, \mathrm{s})$, $4.22(2 \mathrm{H}, \mathrm{s}) 5.23(2 \mathrm{H}, \mathrm{s}), 6.85-6.88(1 \mathrm{H}, \mathrm{d}, J=8.4 \mathrm{~Hz})$, $7.12(1 \mathrm{H}, \mathrm{s}), 7.79-7.82(1 \mathrm{H}, \mathrm{d}, J=8.4 \mathrm{~Hz}), 8.05(1 \mathrm{H}$, s). ${ }^{13} \mathrm{C} \mathrm{NMR}\left(75 \mathrm{MHz}, \mathrm{CDCl}_{3}\right): \delta(\mathrm{ppm}) 25.1,26.2$, 32.9, 53.5, 106.2, 108.6, 117.1, 117.1, 120.2, 125.7, 127.0, 133.2, 138.9, 145.9, 146.1, 166.9, 173.1. FAB mass: $452.83(\mathrm{M}+1)$. Anal. Calcd. for $\mathrm{C}_{17} \mathrm{H}_{14} \mathrm{Br}_{2} \mathrm{~N}_{2} \mathrm{O}_{3}$ : C, 44.96; H, 3.11; N, 6.17. Found: C, 44.97; H, 3.10; N, 6.187 .

2.6a 1',2'-diethyl 5-(4-cyano-1,2-bis(ethoxycarbonyl)-2, 3-dihydro-1H-pyrazol-3-yl)-1-methyl-2-oxospiro[indo-

Table 1. Synthesis of bis-MBH adduct $3 \mathbf{a}$ from mMBH adduct 1a. ${ }^{\text {a }}$

\begin{tabular}{|c|c|c|c|}
\hline & $+\int_{2 a}^{C N}$ & & 1 \\
\hline Entry & Solvents & Time (d) & Yield 3a $(\%)^{\mathrm{b}}$ \\
\hline 1 & $\mathrm{MeOH}$ & 4 & 12 \\
\hline 2 & $\mathrm{CH}_{3} \mathrm{CN}$ & 4 & NR \\
\hline 3 & 1,4-Dioxane & 4 & NR \\
\hline 4 & THF & 4 & trace \\
\hline 5 & DMF & 4 & NR \\
\hline 6 & $\mathrm{CH}_{2} \mathrm{Cl}_{2}$ & 4 & NR \\
\hline 7 & $\mathrm{CHCl}_{3}$ & 4 & NR \\
\hline 8 & $\mathrm{CCl}_{4}$ & 4 & NR \\
\hline 9 & $\mathrm{H}_{2} \mathrm{O}$ & 4 & NR \\
\hline 10 & $\mathrm{MeOH}-\mathrm{H}_{2} \mathrm{O}(1: 1)$ & 4 & NR \\
\hline 11 & $\mathrm{CH}_{3} \mathrm{CN}-\mathrm{H}_{2} \mathrm{O}(1: 1)$ & 4 & NR \\
\hline 12 & 1,4-Dioxane- $\mathrm{H}_{2} \mathrm{O}(1: 1)$ & 4 & 13 \\
\hline 13 & Neat & $8 \mathbf{h}$ & 87 \\
\hline
\end{tabular}

${ }^{\mathrm{a}}$ General condition: $\mathrm{mMBH}$ adduct $\mathbf{1 a}(100 \mathrm{mg}, 0.413 \mathrm{mmol})$, acrylonitrile $2 \mathbf{2 a}$ (1.5 equiv.) and DABCO (20 mol\%) at rt; ${ }^{b}$ Isolated yield; NR-No reaction; d-days. 
line-3,3'-pyrazole ]-1',2',4'-tricarboxylate-methyl-2-oxospiro[indoline-3,3'-pyrazole]-1',2',4'-tricarboxylate (7): Gummy matter, yield $82 \%$. IR $\left(\mathrm{CH}_{2} \mathrm{Cl}_{2}\right)\left(v_{\max }, \mathrm{cm}^{-1}\right)$ : 1609, 1654, 1711, 1720, 2260, 3150. ${ }^{1} \mathrm{H}$ NMR (300 MHz, $\left.\mathrm{CDCl}_{3}\right): \delta(\mathrm{ppm}) 1.25-1.39(12 \mathrm{H}, \mathrm{m}), 3.28$ $(3 \mathrm{H}, \mathrm{s}), 3.59(3 \mathrm{H}, \mathrm{s}), 4.12-4.36(8 \mathrm{H}, \mathrm{m}), 5.96-5.96$
$(1 \mathrm{H}, \mathrm{d}, J=2.5 \mathrm{~Hz}), 6.87-6.89(1 \mathrm{H}, \mathrm{m}), 7.14-7.15(1 \mathrm{H}$, m), 7.32-7.33 (1H, m), 7.34-7.35 (1H, m), 7.40-7.50 $(1 \mathrm{H}, \mathrm{m}) .{ }^{13} \mathrm{C}$ NMR $\left(75 \mathrm{MHz}, \mathrm{CDCl}_{3}\right): \delta(\mathrm{ppm}) 13.9$, 14.2 (2C), 14.9, 26.9, 29.6, 51.8, 64.0, 64.2, 64.2, 64.3, 67.3, 73.7, 108.7, 113.2, 113.5, 121.3, 121.9, 127.8, $131.90,138.7,139.2,144.2,150.9,154.0,157.2$, 164.0,

Table 2. Synthesis of $N$-substituted bis-MBH adducts (3b-g). ${ }^{\text {a }}$

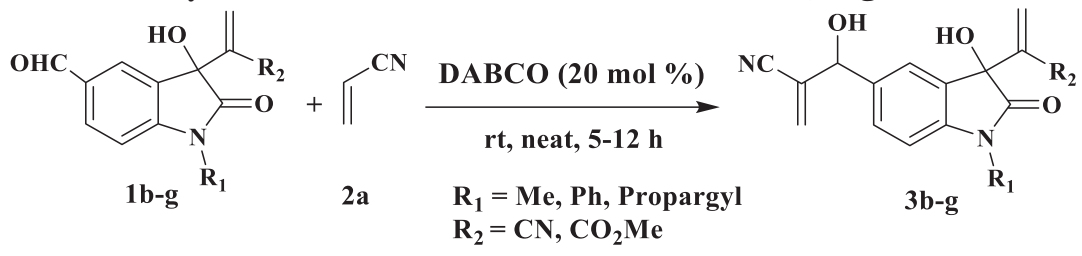

Entry substrates (1)-g)

Time (h) $\quad$ Yield $(\%)^{\mathrm{b}}$

5

87<smiles>C=C(C#N)C(O)c1ccc2c(c1)C(O)(C(=C)C#N)C(=O)N2C</smiles>

6<smiles>C#CCN1C(=O)C(O)(C(=C)C#N)c2cc(C=O)ccc21</smiles>

$1 \mathrm{c}$<smiles>C#CCN1C(=O)C(O)(C(=C)C(C)=O)c2cc(C=O)ccc21</smiles>

12

83<smiles>C#CCN1C(=O)C(O)(C(=C)C)c2cc(C(O)C(=C)C#N)ccc21</smiles>

$1 d$<smiles>C=C(C)C(O)(C(=O)N(Cc1ccccc1)C(C)C)c1cccc(C=O)c1</smiles>

$1 \mathrm{e}$<smiles>C=C(C(C)=O)C1(O)C(=O)N(Cc2ccccc2)c2ccc(C=O)cc21</smiles>

11

88<smiles>C#CCN1C(=O)C(O)(C(=C)C(C)=O)c2cc(C(O)C(=C)C#N)ccc21</smiles>

5

79<smiles>C=C(C#N)C(O)c1ccc2c(c1)C(O)(C(=C)C)C(=O)N2Cc1ccccc1</smiles><smiles>C=C(C#N)C(O)c1ccc2c(c1)C(O)(C(=C)C(C)=O)C(=O)N2Cc1ccccc1</smiles>

$1 f$<smiles>C=C(OC)C(O)(C(=O)OC)C1(O)C(=O)c2cc(C=O)ccc2N1C(C)=O</smiles>

$\lg$<smiles>C=C(C#N)C(O)c1ccc2c(c1)C(O)(C(=C)C(C)=O)C(=O)N2C(C)=O</smiles>

$3 g^{c}$

${ }^{\mathrm{a}}$ General condition: mMBH adduct $\mathbf{1 b}-\mathbf{g}(100 \mathrm{mg})$, acrylonitrile (1.5 equiv.), DABCO $(20 \mathrm{~mol} \%)$, rt; ${ }^{\mathrm{b}}$ Isolated yield; ${ }^{\mathrm{c}}$ Reaction was monitored in 4 days. 
164.2, 164.2, 172.6. FAB mass: $641.54(\mathrm{M}+1)$. Anal. The reaction mixture afforded a bis-MBH adduct 3a Calcd. for $\mathrm{C}_{29} \mathrm{H}_{32} \mathrm{~N}_{6} \mathrm{O}_{11}: \mathrm{C}, 54.37 ; \mathrm{H}, 5.03 ; \mathrm{N}, 13.12$. in $12 \%$ yield after 4 days. The compound 3a was Found: C, 54.39; H, 5.02; N, 13.11. completely characterized by general spectroscopic methods (IR, ${ }^{1} \mathrm{H},{ }^{13} \mathrm{C}$ NMR and mass) (In the ${ }^{1} \mathrm{H}$ NMR spectrum of $\mathbf{3 a}$, the characteristic methine proton of second $\mathrm{MBH}$ adduct appeared at $5.33 \mathrm{ppm}$ ). Encouraged by the preliminary result obtained, the conditions were then optimized for the second $\mathrm{MBH}$ reaction. In view of this,

At the outset, the preliminary reaction was carried out between $\mathrm{mMBH}$ adduct 1a, acrylonitrile $\mathbf{2 a}$ (1.5 equiv.) and $\mathrm{DABCO}(20 \mathrm{~mol} \%)$ in methanol at room temperature. we turned our attention to study the effect of various solvents, solvent-free condition and catalyst load for the

Table 3. Synthesis of bis-functionalized oxindole derivatives (3h-m) ${ }^{\mathrm{a}}$

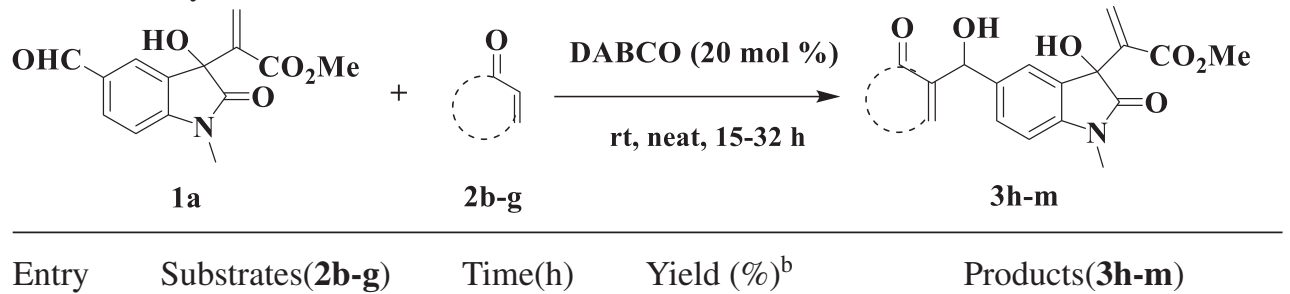

Entry Substrates(2) Time(h) Yield (\%)

${ }^{\mathrm{a}}$ General condition: $\mathrm{mMBH}$ adduct $\mathbf{1 a}(100 \mathrm{mg}, 0.363 \mathrm{mmol})$, activated olefins $\mathbf{2 b - g}(1.5$ equiv.), DABCO (20 mol\%), rt; ${ }^{b}$ Isolated yield. 
second $\mathrm{MBH}$ reaction. The results are summarized in table 1 (entries 1-13).

The catalyst lode was increased more than $20 \mathrm{~mol} \%$ because it did not improve the yield of the product. ${ }^{13}$ To establish a suitable solvent system for the reaction of 1a with 2a, we screened a number of solvents such as methanol, acetonitrile, 1,4-dioxane, tetrahydrofuran, dimethylformamide, dicholoromethane, chloroform, tetrachloromethane and water, etc. (table 1, entries 1-9). Of these, methanol provided bis-MBH adduct in $12 \%$ yield (table 1, entry 1). Binary solvents were reluctant for bis-MBH reaction (entries 9-11). Remarkably, the mixture of 1,4-dioxane:water gave the desired product 3a in low yield (table 1, entry 12). Among them, the neat reaction showed promise for the second $\mathrm{MBH}$ reaction up to $87 \%$ yield (table 1, entry 13 ).

Next, we examined the substrate scope and viability of the second $\mathrm{MBH}$ reaction with a broad range of nitrogen substituted mMBH adducts $\mathbf{1 b}$-f with acrylonitrile under the optimized reaction condition. The results are summarized in table 2.

More interestingly, the reaction progressed well in oxindole nitrogen-bearing electron-donating substituents such as methyl, propargyl and benzyl mMBH adducts and offered the corresponding bis-MBH adducts $\mathbf{3 b}-\mathbf{f}$ in good yields. The reaction was found to be more compatible with the nitrile appended $\mathrm{mMBH}$ adduct than the ester appended mMBH adduct. However, the reaction did not work with $N$-ester mMBH adduct derivative $1 \mathrm{~g}$, presumably due to poor solubility of $1 \mathrm{~g}$ in acrylonitrile.

We then probed the reaction with other activated alkenes to generalize the scope of the second MBH reaction. It was observed that the activated alkenes had a great influence on the rate of the second $\mathrm{MBH}$ reaction. Under the optimized condition, the bis-MBH adducts (3h-m) were obtained in good-to-excellent yields (54-94\%). The results are shown in table 3.

Activated alkenes such as methyl acrylate and butyl acrylate were worked well with $\mathrm{mMBH}$ adduct 1a and afforded bis-MBH adducts (3h and $\mathbf{3 i}$ ) in 94 and $54 \%$ yields, respectively (table 3, entries 1-2). Similarly, with cyclic enones such as cyclopentenone, cyclohexenone and cycloheptenone, the corresponding bis-MBH adducts were obtained (3k, $\mathbf{3} \mathbf{l}$ and $\mathbf{3 m}$ ) in good-to-excellent yields (table 3, entries 4-6). When methyl vinyl ketone was employed as activated olefin, Rauhut-Currier adduct $\mathbf{3 j}$ was observed predominantly along with a trace of uncharacterized product. ${ }^{14}$ It should be noted that the present work discloses the cyclic enone appended bis-MBH adducts exclusively without prerequisite of any promoters and/or Lewis acids. $^{15}$

The successful accomplishment of bis-MBH adducts prompted us to explore further the functionalization via isomerization with various nucleophilic reagents such as $\mathrm{Ar}-\mathrm{H}, \mathrm{CH}(\mathrm{OMe})_{3}$, propargyl alcohol and $\mathrm{HBr}^{16}{ }^{16}$ The results are summarized in table 4.

Accordingly, bis-MBH adduct $\mathbf{3 b}, \mathrm{K}-10$ clay with benzene or trimethoxymethane underwent isomerization smoothly and afforded the corresponding highly functionalized mono- and bis-allyl derivative in good yields (table 3, entries 1-2). However, with propargyl alcohol, mono-isomerized compound $\mathbf{4} \mathbf{c}$ was exclusively obtained from bis-MBH adduct $\mathbf{3 b}$ under solvolysis condition (table 3 , entry 3 ). Under the microwave irradiation, mono- and bis-bromo allyl derivatives were obtained in good yield from $\mathrm{mMBH}$ adduct $\mathbf{3 b}$ with aqua. $\mathrm{HBr}$ (table 3, entry 4). A notable trend was observed that isomerization preferentially takes place at the $\mathrm{C}-5$ position $\left(2^{\circ}\right.$-alcohol) rather than at the $\mathrm{C}-3$ position $\left(3^{\circ}\right.$ alcohol) (table 3, entries 1-4). The novel mono- and

Table 4. Synthesis of mono- and bis-functionalized oxindole derivatives $(\mathbf{4} \& \mathbf{5}){ }^{\mathrm{a}}$

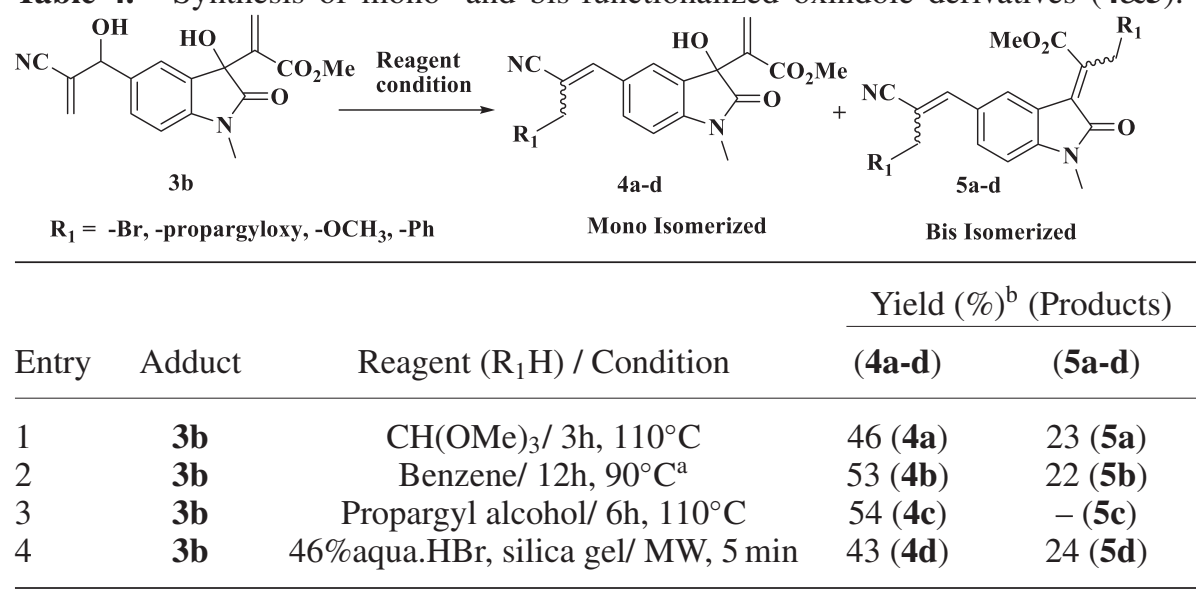

${ }^{\mathrm{a}} 50 \%$ w/w Mont. K-10 clay was used as catalyst; ${ }^{\mathrm{b}}$ Isolated yield. 

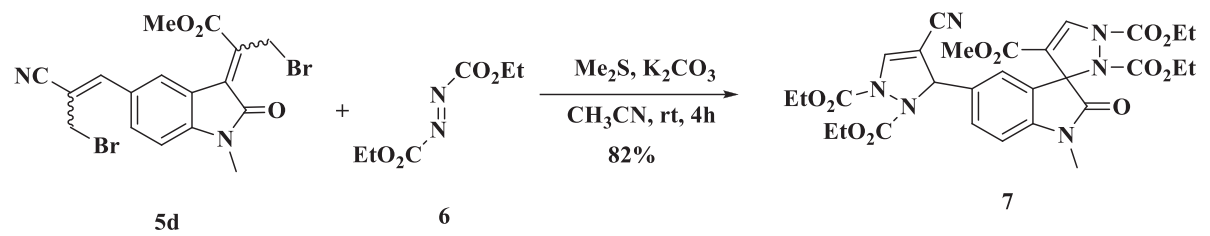

Scheme 1. Synthesis of bis-pyrazole derivative 7 from bis-allyl bromides of oxindole.

bis-isomerized compounds were unambiguously characterized by various spectroscopic techniques.

Furthermore, synthetic utility was demonstrated by the synthesis of medicinally important bis-pyrazole derivative (7) and was prepared from 5d, diethyl diazenedicarboxylate $\mathbf{6}, \mathrm{Me}_{2} \mathrm{~S}$ and $\mathrm{K}_{2} \mathrm{CO}_{3}$ exploiting $[3+2]$-annulation reaction (scheme 1). ${ }^{17,18}$

All the new compounds were thoroughly characterized by spectroscopic methods (IR, ${ }^{1} \mathrm{H},{ }^{13} \mathrm{C}$ NMR and FAB mass spectra).

\section{Conclusions}

An efficient synthesis of bis-functionalized oxindole derivatives from 3, 5-bis-MBH adducts of isatin via isomerization is disclosed for the first time. The 5-formyl $\mathrm{MBH}$ adduct of oxindole has displayed a wide substrate scope, rate acceleration and selective formation of bis$\mathrm{MBH}$ adduct with cyclic enones with DABCO as catalyst. The synthetic utility of bis-allyl derivative was demonstrated by the synthesis of potent bis-pyrazole derivative via [3+2]-annulation strategy.

\section{Supplementary Information}

The electronic supplementary information consists of the general procedure for the synthesis of target molecules and their analytical data/spectral proof in detail. Supplementary Information is available at www. ias.ac.in/chemsci.

\section{Acknowledgements}

KS thanks Thiagarajar College, Madurai; KAP thanks Kamaraj College, Tuticorin; and PS thank Tamilnadu Open University, Chennai, for providing lab facilities. RVLV thanks director of NIIST for providing infrastructure facilities. Thanks are due to Mrs. Viji and Mrs. Saumini Mathew for providing Mass and NMR spectra.

\section{References}

1. (a) Alcaide $\mathrm{B}$, Almendros $\mathrm{P}$ and Rodríguez-Acebes $\mathrm{R}$ 2005 Chem. Eur. J. 11 5708; (b) Cui C B, Kakeya H and Osada H 1996 Tetrahedron 52 12651; (c) Xue J,
Zhang Y, Wang X I, Fun H K and Xu J H 2000 Org. Lett. 2 2583; (d) Klumpp D A, Yeung K Y, Prakash G K S and Olah G A 1998 J. Org. Chem. 63 4481; (e) Ciganek E 1997 In Organic Reactions L A Paquette (ed.) vol. 51 (New York: John Wiley) pp. 201-359; (f) Langer P 2000 Angew. Chem. Int. Ed. 39 3049; (g) Da Silva J F M, Garden S J and Pinto A C $2001 \mathrm{~J}$. Braz. Chem. Soc. 12273

2. (a) Booker-Milburn K I, Fedouloff M, Paknoham S J, Strachan J B, Melville J L and Voyle M 2000 Tetrahedron Lett. 41 4657; (b) Tratrat C, Giorgi-Renault S and Husson H P 2000 J. Org. Chem. 65 6773; (c) Wang L, Zhang Y, Hu H Y, Fun H K and Xu J H 2005 J. Org. Chem. 70 3850; (d) Alcaide B, Almendros P, Del Campo T M and Carrascosa R 2008 Chem. Asian. J. 31140

3. (a) Coppola G M 1987 J. Heterocycl. Chem. 24 1249; (b) Zawadowka I 1975 Acta Pol. Pharm. 32 33; (c) Black D S C, Bowyer M C, Catalano M M, Ivory A J, Keller P A, Kumar N and Nugent S J 1994 Tetrahedron 5010497

4. (a) Tomchin A B and Tumanova I V 1990 Org. Lett. 26 1327; (b) Gasparic J, Vontor T, Lycka A and Snobl D 1990 Chem. Commun. 55 2963; (c) Martinez F and Narmann H 1990 Synth. Met. 3 9195; (d) Jnaneswar G K and Deshpande V H 1999 J. Chem. Res. 632

5. Mason J J, Janosik T and Bergman J 2009 Synthesis 21 3642

6. (a) Liu Y L, Wang B L, Cao J J, Chen L, Zhang Y X, Wang C and Zhou J 2010 J. Am. Chem. Soc. 132 15176; (b) Garden S J and Skakleb J M S 2002 Tetrahedron Lett. 43 1972; (c) Tsuda M, Kasai Y, Komatsu K, Sone T M, Tanaka Y and Mikami Kobayashi J 2004 Org. Lett. 6 3087; (d) Mugishima T, Tsuda M, Kasai Y, Ishiyama H, Fukushi E, Kawabata J, Watanabe M, Akao K and Kobayashi J 2005 J. Org. Chem. 709430

7. (a) Peng J, Huang X, Cui H L and Chen Y C 2010 Org. Lett. 12 4260; (b) Alcaide B, Almendros P and Rodriguez-Acebes R 2006 J. Org. Chem. 712346

8. (a) Shanmugam P Vaithiyanathan V and Selvakumar K 2008 Tetrahedron Lett. 49 2119; (b) Zhang Y and Panek, J S 2009 Org. Lett. 11 3366; (c) Sebahar P R and Williams R M 2000 J. Am. Chem. Soc. 122 5666; (d) Lerchner A and Carreira E M 2006 Chem. Eur. J. 12 8208; (e) Nag S, Pathak R, Kumar M, Shuklacv P K and Batra S 2006 Bioorg. Med. Chem. Lett. 16 3824; (f) Lei X and Porco J A 2006 J. Am. Chem. Soc. 128 14790; (g) Alcaide B, Almendros P, del Campo T M and Quiros T M 2009 Chem. Eur. J. 15 3344; (h) Niu Q, Mao H, Yuan G, Gao J, Liu H, Tu Y, Wang X and Lv X 2013 Adv. Synth. Catal. 355 1185; (i) Selvakumar K, Lingam K A P and Varma R V L 2014 RSC. Adv. 436538

9. (a) Basavaiah D, Rao A J and Satyanarayana T 2003 Chem. Rev. 103 811; (b) Divya K N, Shaikh M, Mobin, Irishi N N and Namboothiri 2012 Org. Lett. 14 4580; 
(c) Basavaiah D and Gorre Veeraraghavaiah 2012 Chem. Soc. Rev. 41 68; (d) Singh G S and Desta Z Y 2012 Chem. Rev. 1126104

10. (a) Aggarwal V K and Mereu A 1999 Chem. Commun. 2311; (b) Roos G H P and Rampersadh P 1993 Synth. Commun. 23 1261; (c) Kundu M K, Mukherjee S B, Balu N, Padmakumar R and Bhat S V 1994 Synlett 444; (d) Hayashi Y, Okada K, Ashimine I and Shoji M 2002 Tetrahedron Lett. 43 8683; (e) Rafel S and Leahy J W 1997 J. Org. Chem. 62 1521; (f) Kawamura M and Kobayashi S 1999 Tetrahedron Lett. 40 1539; (g) Aggarval V K, Mereu A, Farver G J and McCague R 1998 J. Org. Chem. 63, 7183; (h) Shi M, Jiang J K and Feng Y S 2000 Org. Lett. 22397

11. (a) Ciganek E 1997 In Organic Reactions L A Paquette (ed.) vol. 51 (New York: John Wiley) pp. 201-359; (b) Basavaiah D, Rao K V and Reddy R J 2007 Chem. Soc. Rev. 36 1581; (c) Singh V and Batra S 2008 Tetrahedron 644511
12. (a) Basavaiah D, Muthukumaran K and Sreenivasulu B 2000 Synthesis 545; (b) Basavaiah D, Padmaja K and Satyanarayana T 2000 Synthesis 1662

13. Yu C, Liu B and Hu L 2001 J. Org. Chem. 665413

14. Ma G N, Jiang J J, Shi M and Weib Y 2009 Chem. Commun. 5496

15. Bugarin A and Connell B T 2009 J. Org. Chem. 744638

16. (a) Shanmugam $\mathrm{P}$ and Rajasingh $\mathrm{P} 2002$ Chem. Lett. 1212; (b) Shanmugam P and Viswambharan B 2008 Synlett. 2763

17. (a) Goodell J R, Puig-Basagoiti F, Forshey B M, Shi P Y and Ferguson D M 2006 J. Med. Chem. 49 2127; (b) Manna F, Chimenti F, Fioravanti R, Bolasco A, Secci D, Chimenti P, Ferlini C and Scambia G 2005 Bioorg. Med. Chem. Lett. 15 4632; (c) Bekhit A A and Abdel-Aziem T 2004 Bioorg. Med. Chem. 12 1935

18. Selvakumar K, Vaithiyanathan $\mathrm{V}$ and Shanmugam $\mathrm{P}$ 2010 Chem. Commun. 2826 